\title{
Simulating chiral quarks in the $\epsilon$-regime of QCD
}

\author{
W. Bietenholz ${ }^{\text {a }}$, T. Chiarappa ${ }^{\text {b }}$, K. Jansen ${ }^{\text {b }}$, K.-I. Nagai ${ }^{\text {b }}$ and S. Shcheredin ${ }^{\text {a }}$

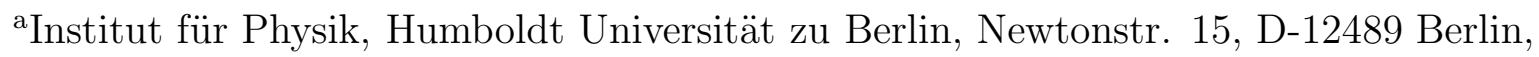 \\ Germany \\ b NIC/DESY Zeuthen, Platanenallee 6, D-15738 Zeuthen, Germany
}

We present simulation results for lattice QCD with chiral fermions in small volumes, where the $\epsilon$-expansion of chiral perturbation theory $(\chi \mathrm{PT})$ applies. Our data for the low lying Dirac eigenvalues, as well as mesonic correlation functions, are in agreement with analytical predictions. This allows us to extract values for the leading Low Energy Constants $F_{\pi}$ and $\Sigma$.

\section{The $\epsilon$-regime of QCD}

For QCD at low energy and zero quark masses one assumes the chiral symmetry to break spontaneously, $S U\left(N_{f}\right)_{L} \otimes S U\left(N_{f}\right)_{R} \rightarrow S U\left(N_{f}\right)_{L+R}$. This yields $N_{f}^{2}-1$ NambuGoldstone bosons, which pick up a small mass when we proceed to light quark masses $m_{q}$ (we assume the same $m_{q}$ for all flavours involved). These bosons are identified with the light mesons, in particular the pions (and often also the kaons and $\eta$ ).

$\chi \mathrm{PT}$ is a powerful description for them by means of an effective chiral Lagrangian,

$\mathcal{L}_{\text {eff }}[U]=\frac{F_{\pi}^{2}}{4} \operatorname{Tr}\left[\partial_{\mu} U \partial_{\mu} U^{\dagger}\right]-\frac{m_{q}}{2} \Sigma \operatorname{Tr}\left[U+U^{\dagger}\right]+\ldots$,

which deals with fields $U$ in the coset space $S U\left(N_{f}\right)$ of the chiral symmetry breaking. One writes down the terms which fulfil the symmetry requirements and classifies them according to low energy counting rules. The coefficients to these terms are the Low Energy Constants (LEC), hence $F_{\pi}$ and $\Sigma$ are the leading LEC.

One usually considers this effective theory in an infinite or very large volume, which allows for an expansion in the meson momenta ( $p$-expansion). However, one can also consider the opposite situation — a small volume with linear size $L \ll 1 / m_{\pi}$ — which is called the $\epsilon$-regime. Here the $p$-expansion fails due to the importance of the zero modes. Fortunately the path integral for the latter can be performed with collective variables [ 1. Then an expansion in the low (non-zero) momenta and the meson mass is possible ( $\epsilon$-expansion).

It is in fact motivated to study the $\epsilon$-regime, although it is not a physical situation to squeeze pions into such a tiny box. The point is that even in a small volume values for the LEC in infinite volume can be determined - exactly by analysing the finite size effects.

The LEC enter the effective Lagrangian as free parameters, hence they can only be determined from QCD as the fundamental theory (or from experiment). This is a goal for lattice simulations, which are clearly more convenient in small volumes. 
However, simulations in the $\epsilon$-regime face technical difficulties, which could only be overcome in the recent years. One has to keep track of the chiral symmetry and deal with very light pions. Moreover, we need a clean definition for the topology, since expectation values should often be measured in distinct topological sectors [ 2]. These properties are provided by Ginsparg-Wilson fermions, which have a lattice modified, exact chiral symmetry [3] and exact zero modes, so that the topological charge can be defined as the index $\nu$ (i.e. the difference between the number of zero modes with positive and negative chirality) [4]. The simplest realization of a Ginsparg-Wilson fermion uses the overlap Dirac operator [5].

Although algorithmic tools for the $\epsilon$-regime have been worked out [ [6], such simulations are still computationally expensive: for the time being one has to use the quenched approximation — which leads to logarithmic finite size effects on the LEC [ [].

\section{The Dirac spectrum}

Chiral Random Matrix Theory (RMT) simplifies QCD to a Gaussian distribution of fermion matrix elements, and still manages to capture some aspects of QCD. One of its numerous assumptions is that the energy should be well below the Thouless energy $E_{\text {Thouless }}=F_{\pi}^{2} /(\sqrt{V} \Sigma)$.

In particular, Ref. [8] presents explicit predictions for the microscopic spectral density $\rho_{s}(z)=\left.\frac{1}{\Sigma V} \rho\left(\frac{z}{\Sigma V}\right)\right|_{V \rightarrow \infty}=\sum_{\nu=0}^{\infty} \rho_{s}^{(|\nu|)}(z), z=\lambda \Sigma V, \lambda:$ eigenvalue of the Dirac operator.

The density in each topological sector $|\nu|$ is further decomposed into densities of the leading individual eigenvalues, $\rho_{s}^{(|\nu|)}(z)=\sum_{n=1,2,3 \ldots} \rho_{n}^{(|\nu|)}(z)$. Using the overlap operator at $m_{q}=0$ we have measured $\rho_{1}^{(|\nu|)}(z)$ at $|\nu|=0,1$ and 2 in different volumes [9]. In Figure 1 we compare our data for the cumulative density with the RMT predictions. The comparison fails in the small volume $V \simeq(0.98 \mathrm{fm})^{4}$, but it works well in $V \simeq(1.23 \mathrm{fm})^{4}$. In this fit, $\Sigma$ appears as the only free parameter. The successful fit in $V \simeq(1.23 \mathrm{fm})^{4}$ implies a value of $\Sigma \simeq(253 \mathrm{MeV})^{3}$, which agrees well with the literature.

The microscopic RMT predictions work in such volumes up to the second eigenvalue, then the spectral density starts to grow cubically (bulk behaviour). This transition can be viewed as an effective bound for the chiral RMT, thus taking the rôle of $E_{\text {Thouless }}$. Our observations were confirmed by subsequent studies, which included larger volumes [10].

\section{Axial vector correlation functions}

Since QCD simulations with chiral fermions are restricted to the quenched approximation at present, also quenched $\chi \mathrm{PT}$ was worked out [11] as a basis of predictions that the lattice data can be confronted with. Regarding the mesonic correlation functions in leading order, the vector correlator vanishes (actually to all orders), and the scalar and pseudoscalar correlators have the drawback that they involve additional LEC, which are specific to quenching. Hence we considered the correlator of the axial current $A_{\mu}(t)=\sum_{\vec{x}} \Psi(t, \vec{x}) \gamma_{5} \gamma_{\mu} \Psi(t, \vec{x})$, where the leading order only involves $F_{\pi}$ and $\Sigma$. The $\epsilon-$ regime prediction for $\left\langle A_{0}(t) A_{0}(0)\right\rangle_{|\nu|}$ in a volume $L^{3} \times T$ is a parabola in $t$ with a minimum at $T / 2$, and with an additive constant $F_{\pi}^{2} / T$ (this is in contrast to the usual cosh behaviour in large volumes). The curvature in the minimum of the parabola is $\propto \Sigma$. We now 

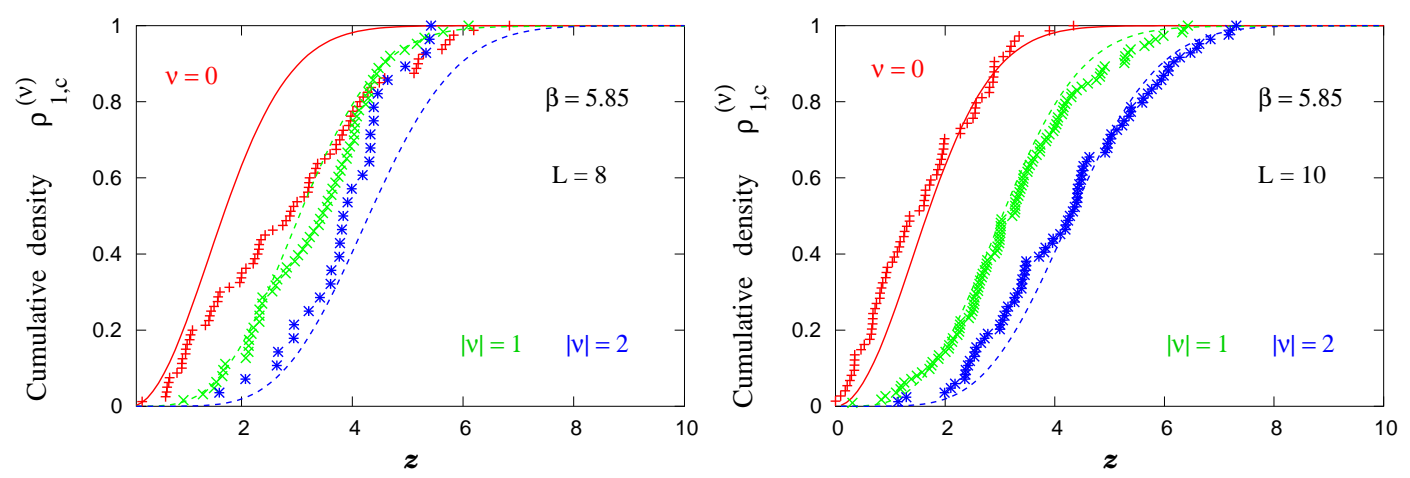

Figure 1. The cumulative density of the first non-zero eigenvalue in the sectors $|\nu|=0,1$ and 2. The comparison of our lattice data to the chiral RMT predictions (curves) fails in $V=(0.98 \mathrm{fm})^{4}$ (on the left), but it is successful in $V=(1.23 \mathrm{fm})^{4}$ (on the right). In the latter case, the fit yields $\Sigma=(253 \mathrm{MeV})^{3}$.

simulated overlap fermions at $m_{q}=21.3 \mathrm{MeV}$ [12]. A first experiment with $L \simeq 0.98 \mathrm{fm}$ failed to reproduce the prediction. This is exactly consistent with the observation for the Dirac eigenvalues described before: in both cases we badly need $L \gtrsim 1.1 \mathrm{fm}$.

We also observed that the Monte Carlo history at $\nu=0$ is plagued by spikes, so that a huge statistics is needed. These spikes occur at the configurations which have a very small (non-zero) Dirac eigenvalue. As Figure 1 shows, this probability is strongly suppressed for $|\nu|>0$, and indeed these histories are smoother.

So we measured in a volume $V=(1.12 \mathrm{fm})^{4}$ at $|\nu|=1$. Figure 2 (on the left) shows that we found a nice agreement with the predicted parabola, which is plotted for $\Sigma=0$ and for $\Sigma=(250 \mathrm{MeV})^{3}$. Since these curves can hardly be distinguished, we see that the sensitivity of the curvature is by far too small for a determination of $\Sigma$ in this way. On the other hand, this result provides a stable value of $F_{\pi}=(86.7 \pm 4.0) \mathrm{MeV}$. The plot on the right-hand-side of Figure 2 shows the value we obtained as a function of the fitting range around the minimum. The result that we obtained for $|\nu|=2$ (with lower statistics) agrees within the errors. The renormalisation due to the factor $Z_{A}$ drives our result for $F_{\pi}$ up to a value around $130 \mathrm{MeV}$, but it is well-known that quenched results for the pion decay constant tend to be significantly above the experimental value of $\approx 93 \mathrm{MeV}$ resp. $\approx 86 \mathrm{MeV}$ in the chiral limit [13].
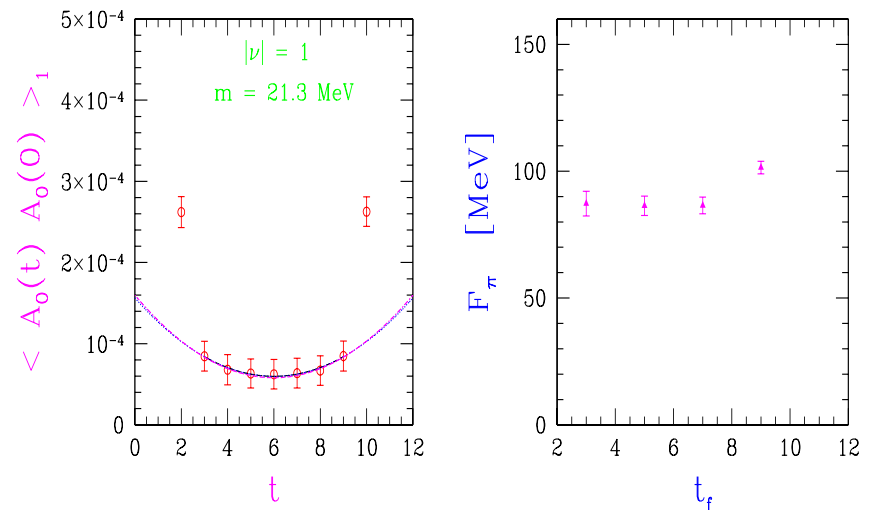

Figure 2. The axial correlation function (on the left) and the values of $F_{\pi}$ obtained from fits over $t_{f}$ points around the centre $t=6$ (on the right). 


\section{Conclusions}

We have performed two pilot studies about the feasibility to determine LEC of $\chi \mathrm{PT}$ from simulations with chiral lattice fermions in the $\epsilon$-regime. Our results show that such lattice data can be fitted to the prediction by chiral RMT and quenched $\chi \mathrm{PT}$. However, we also found crucial conditions for this to work: the spatial box length must exceed a lower bound, $L \gtrsim 1.1 \mathrm{fm}$. Moreover, correlators should not be measured in the topologically trivial sector.

$F_{\pi}$ can be extracted from the axial vector correlator, and $\Sigma$ from the densities of the lowest eigenvalues of the Dirac operator. Thus our pilot studies confirm that simulations in the $\epsilon$-regime do have the potential to evaluate LEC from first principles. One could also deal solely with the zero-mode contributions to the mesonic correlation functions [ 14 .

Currently a non-standard lattice gauge action is being tested, which preserves the topological charge over long intervals of the Monte Carlo history (far beyond the autocorrelation time of other observables) [15]. This would allow us to collect many configurations for measurements in a specific topological sector, which is tedious with the standard gauge action. On the fermionic side, we are now including an improved overlap operator [16] in our investigation of the $\epsilon$-regime. At last we add that we also have results in the $p$-regime [17, for instance for the direct measurement of $F_{\pi}$.

This work was supported by the Deutsche Forschungsgemeinschaft through SFB/TR903. Most computations were performed at the ZIB/HLRN in Berlin.

\section{REFERENCES}

1. J. Gasser and H. Leutwyler, Phys. Lett. B188 (1987) 477.

2. H. Leutwyler and A. Smilga, Phys. Rev. D46 (1992) 5607.

3. M. Lüscher, Phys. Lett. B428 (1998) 342.

4. P. Hasenfratz, V. Laliena and F. Niedermayer, Phys. Lett. B427 (1998) 317.

5. H. Neuberger, Phys. Lett. B417 (1998) 141.

6. L. Giusti, C. Hoelbling, M. Lüscher and H. Wittig, Comput. Phys. Commun. 153 (2003) 31.

7. P.H. Damgaard, Phys. Lett. B608 (2001) 162.

8. P.H. Damgaard and S.M. Nishigaki, Nucl. Phys. B518 (1998) 495; Phys. Rev. D63 (2001) 045012.

9. W. Bietenholz, K. Jansen and S. Shcheredin, JHEP 07 (2003) 033.

10. L. Giusti, M. Lüscher, P. Weisz and H. Wittig, JHEP 11 (2003) 023. D. Galletly et al., Nucl. Phys. (Proc. Suppl.) B129/130 (2004) 456.

11. P.H. Damgaard et al., Nucl. Phys. B629 (2002) 226; Nucl. Phys. B656 (2003) 226.

12. W. Bietenholz, T. Chiarappa, K. Jansen, K.-I. Nagai and S. Shcheredin, JHEP 02 (2004) 023.

13. G. Colangelo and S. Dürr, Eur. Phys. J. C33 (2004) 543.

14. L. Giusti, P. Hernández, M. Laine, P. Weisz and H. Wittig, JHEP 0401 (2004) 003. S. Shcheredin, Ph.D. thesis, Berlin, 2004.

15. H. Fukaya and T. Onogi, Phys. Rev. D68 (2003) 074503; Phys. Rev. D70 (2004) 054508. W. Bietenholz, K. Jansen, K.-I. Nagai, S. Necco, L. Scorzato and S. Shcheredin, hep-lat/0409073; hep-lat/0412017.

16. W. Bietenholz, Eur. Phys. J. C6 (1999) 537; Nucl. Phys. B644 (2002) 223. 
17. $\chi$ LF collaboration, JHEP 0412 (2004) 044. 